\title{
Calidad de carne y rendimiento a la faena de pollos parrilleros híbridos machos criados en un sistema de producción aviar alternativo
}

\author{
Arbizu, J.M.'; Fernández, R. ${ }^{2}$; Sanz, P. ${ }^{2}$; Sindik, M. ${ }^{2}$; Rébak, G. ${ }^{3}$ \\ ${ }^{1}$ Cát. Aliment. Nutr. Animal, Instituto Universitario, Formosa (Argentina). \\ ${ }^{2}$ Cát. Prod. Aves, Fac.Cs.Vet. UNNE, Corrientes (Argentina) ${ }^{3}$ Cát.Tecn. Alim., Fac.Cs.Vet. UNNE. \\ E-mail: flacarbizu@hotmail.com
}

\begin{abstract}
Resumen
Arbizu, J.M.; Fernández, R.; Sanz, P.; Sindik, M.; Rébak, G.: Calidad de carne y rendimiento a la faena de pollos parrilleros hibridos machos criados en un sistema de producción aviar alternativo. Rev. Vet. 32: 2, 151-154, 2021. La producción avícola alternativa está creciendo debido a la demanda del consumidor para productos diferenciados, como la carne de aves criadas en sistemas pastoriles. El objetivo de este trabajo fue evaluar el efecto de la utilización de un sistema semi-extensivo con acceso a pastura $(\mathrm{T})$ sobre el rendimiento a la faena y calidad de la carne en pollos Cobb 500 machos, en un ciclo de 49 días, comparado con un sistema en confinamiento (C), en las diferentes estaciones del año. Se realizó análisis estadístico mediante ANOVA para un diseño factorial $2 \times 4$ (2 sistemas de crianza y 4 estaciones). Se observaron diferencias estadísticamente significativas para variables de calidad de carne con valores de pérdidas por cocción ( $\mathrm{PPC}, \mathrm{p}=0,019) 26,68 \pm 1,63$ (C), 29,39 $\pm 1,39(\mathrm{~T}) \%, \mathrm{pH}(\mathrm{p}=0,039), 5,94 \pm 0,04(\mathrm{C}), 6,02 \pm 0,03(\mathrm{~T})$, sugiriendo un diferente equilibrio energético a favor de los pollos del grupo control. Por su parte la terneza evidenció diferencias significativas $(\mathrm{p}=0,025) 1,16 \pm 0,06(\mathrm{C}), 1,32 \pm 0,08(\mathrm{~T}) \mathrm{kg} / \mathrm{cm}^{2}$, que permite asumir un efecto de la actividad física sobre el acúmulo de tejido conectivo en los músculos del grupo tratado. En las variables de rendimiento se observaron diferencias significativas con valores de rendimiento de pata-muslo $(\mathrm{p}=0,036) 27,33 \pm 0,2(\mathrm{C}), 27,9 \pm 0,29(\mathrm{~T}) \%$, grasa acumulada ( $\mathrm{p}=<0,0001) 1,49 \pm 0,13(\mathrm{C}), 0,94 \pm 0,08(\mathrm{~T}) \%$, aludiendo que la mayor actividad física desarrollada por el grupo tratado, dio por resultado una merma en la cantidad de grasa abdominal junto a una mayor acreción proteica en pata-muslo. En rendimiento de pechuga $(p=0,031) 31,6 \pm 0,23(\mathrm{C}), 30,8 \pm 0,33(\mathrm{~T}) \%$, los pollos del grupo control distribuyeron en forma diferente los nutrientes suministrados, resultando en mayor desarrollo de pechuga y acumulo graso abdominal. Se concluye que el sistema de alojamiento afecta las características tecnológicas y la composición de la canal, que se reflejan en la diferente distribución de nutrientes mediante modificaciones en la actividad física desarrollada por las aves.
\end{abstract}

Palabras clave: pollos parrilleros, sistema pastoril, calidad, carne, acumulo graso.

\begin{abstract}
Arbizu, J.M.; Fernández, R.; Sanz, P.; Sindik, M.; Rébak, G.: Meat quality and slaughter performance of male hybrid broilers reared in an alternative poultry production system. Rev. Vet. 32: 2, 151-154, 2021. Alternative poultry production is growing due to consumer demand for differentiated products, such as meat from poultry raised in pastoral systems. The objective of this work was to evaluate the effect of the use of a semi-extensive system with access to pasture (T) on slaughter performance and meat quality in male Cobb 500 chickens, in a cycle of 49 days, compared to a system in confinement (C), in the different seasons of the year. Statistical analysis was performed using ANOVA for a $2 \times 4$ factorial design (2 rearing systems and four seasons). Statistically significant differences were observed for meat quality variables with PPC values $(\mathrm{p}=0.019), 26.68 \pm 1.63(\mathrm{C}), 29.39 \pm 1.39(\mathrm{~T}) \%, \mathrm{pH}(\mathrm{p}=0.039)$ $5,94 \pm 0.04$ (C) $6.02 \pm 0.03(\mathrm{~T})$, suggesting a different energy balance in favor of the chickens in the control group. On the other hand, tenderness showed significant differences $(p=0.025)$ $1.16 \pm 0.06(\mathrm{C}), 1.32 \pm 0.08(\mathrm{~T}) \mathrm{kg} / \mathrm{cm}^{2}$, which allows to assume an effect of physical activity on the accumulation of connective tissue in the muscles of the treated group. In the yielding variables, significant differences were observed with yielding values of leg thigh $(\mathrm{p}=0.036)$ $27.33 \pm 0.2(\mathrm{C}), 27.9 \pm 0.29(\mathrm{~T}) \%$, accumulated fat $(\mathrm{p}=<0.0001) 1.49 \pm 0.13(\mathrm{C}), 0.94 \pm 0.08$ (T)\%, alluding that the greater physical activity developed by the treated group resulted in a decrease in the amount of abdominal fat along with a higher protein accretion in leg thigh. In breast yield ( $\mathrm{p}=0.031) 31.6 \pm 0.23$ (C) $30.8 \pm 0.33(\mathrm{~T}) \%$, the chickens in the control group
\end{abstract}


distributed the nutrients supplied differently, resulting in greater breast development and abdominal fat accumulation. It is concluded that the housing system affects the technological characteristics and the composition of the carcass that are reflected in the different distribution of nutrients through modifications in the physical activity developed by the birds.

Key words: chickens grilled, pastoral system, quality, meat, fat accumulate.

\section{INTRODUCCIÓN}

En la Provincia de Formosa, al igual que en los demás departamentos de la región nordeste argentino (NEA), la producción avícola es secundaria para la mayoría de las familias rurales y se caracteriza por una avicultura de traspatio que aprovecha al máximo la mano de obra familiar con instalaciones e implementos de escaso desarrollo, poco dependientes del capital financiero ${ }^{5,15}$

Esta producción de traspatio con aves en libertad, contribuye a mejorar la ingesta de proteínas en la población rural, disminuir la pobreza, empoderar a la mujer y mantener la salud ${ }^{7}$, aunque persiste la necesidad de implementar sistemas alternativos que permitan mejorar los medios de vida de los hogares rurales, proporcionándoles ingresos adicionales ${ }^{12}$.

Los sistemas de producción se clasifican tradicionalmente en extensivos, intensivos e intermedios denominados semi-extensivos y semi-intensivos ${ }^{6,16}$. La avicultura alternativa es básicamente cualquier forma que difiera de la avicultura clásica o convencional. Esta variación se refiere a los sistemas de producción menos intensivos y su objetivo es obtener huevos y carne de aves diferenciadas de los productos de consumo masivo, por su especial calidad y/o el modo de cría ${ }^{2}$.

En estas regiones existen algunos factores que deben ser tenidos en cuenta si se pretende que el potencial productivo existente resuelva los problemas acuciantes de la población. Entre estos aspectos, la disponibilidad de pollitos de un día, en cantidad y calidad, constituye una ventaja estratégica como motor para el desarrollo de la avicultura, que puede ser garantizada mediante el empleo de pollos parrilleros.

La crianza de este genotipo bajo sistemas semiextensivos con acceso a una pastura ${ }^{15}$, representa -en términos de rendimiento a la faena y calidad de la carne- una alternativa productiva viable para los pequeños productores, en tanto posibilita el acceso a un producto de alto valor nutritivo con una oferta regular para la venta, principalmente en las ferias francas, generando así ingresos en la economía familiar.

Estos productos son muy bien aceptados y buscados por el consumidor, pero su limitante es la falta de una oferta regular, como así también otro aspecto a tener en cuenta es la ausencia de estandarización del producto final en cada ciclo de producción ${ }^{3}$.

El objetivo de este trabajo fue evaluar el efecto de la utilización de un sistema semi-extensivo con acceso a pastura, en machos de pollos parrilleros híbridos, sobre el rendimiento a la faena y la calidad de la car- ne, en comparación con un sistema en confinamiento, en las diferentes estaciones del año en la Provincia de Formosa.

\section{MATERIAL Y MÉTODOS}

Los ensayos se realizaron en un establecimiento rural de Loma Tuyuyú, ubicado sobre la Ruta Nacional $86, \mathrm{~km} 1358$, perteneciente a la localidad de Laguna Blanca, región subtropical norte de Formosa.

A tal fin se dispuso de un predio con una superficie aproximada de $332,5 \mathrm{~m}^{2}$ (19 x 17,5 m) sembrado con alfalfa. Allí se construyó un galpón de ventilación natural, con una superficie total de $37,5 \mathrm{~m}^{2}(7,5 \mathrm{~m} \mathrm{x}$ $5 \mathrm{~m}$ ), montado con palmas (Copernicia alba: postes para el armazón y tejas para el techo); con orientación este-oeste atendiendo las características climáticas de la zona.

Fue internamente dividido en 10 boxes de $1,875 \mathrm{~m}^{2}$ de superficie, ubicando 5 a cada lado de un pasillo central. Cada box tuvo acceso a un parque individual de $10,5 \mathrm{~m}^{2}$ de superficie, contando con bebederos y comederos en cada uno de ellos.

Se llevaron a cabo cuatro ensayos, distribuidos a razón de uno por cada estación del año. El tiempo de duración de cada ensayo fue de 49 días. Los registros de las variables productivas se tomaron desde el inicio y cada 7 días hasta el final del ensayo.

Para cada ensayo se utilizó un lote de 150 pollos parrilleros machos (600 en total), que a su llegada al establecimiento fueron divididos al azar en diez boxes (unidades experimentales) de 15 aves cada uno, identificadas individualmente con caravanas numeradas.

Se asignaron cinco boxes al grupo A (control), donde los pollos fueron criados en un sistema intensivo sin acceso a parque durante todo el periodo del ensayo (49 días). En los cinco boxes del grupo B (tratado), las aves tuvieron acceso a parque a partir del día 21 de vida, durante las horas de sol. El trabajo se realizó durante los años 2018 y 2019. Al finalizar el ciclo se sacrificaron tres pollos, elegidos al azar, por cada box, considerándose cada box como una unidad experimental, con un tamaño muestral $n=40$.

Los pollos fueron sacrificados por yugulación, previa insensibilización mediante dislocación cervical ${ }^{10}$, con un ayuno de 6 horas. Posteriormente se procedió al registro de datos y la obtención de muestras, las cuales fueron acondicionadas en bolsas de polietileno de primer uso, identificadas con tarjetas de alto impacto, registrando número de caravana de cada animal según lote y fecha de faena. Las mismas fueron acondiciona- 
das en conservadoras con refrigerante o hielo, para su traslado al laboratorio. Las variables estudiadas en el presente trabajo fueron:

\section{Variables de faena}

Al final del ensayo se registró:

Peso corporal (PC, en g). Se obtuvo mediante balanza digital, inicialmente con proximidad al gramo (hasta la tercera semana) y con aproximación a los $10 \mathrm{~g}$ desde la tercera semana en adelante.

Peso limpio (PL, en g). Incluyó el peso de la canal sin cabeza, patas ni vísceras.

Rendimiento de la canal (RC, en \%). Fue obtenido por cálculo, dividiendo el PL por el $\mathrm{PC}$, multiplicando por 100 .

Rendimiento de carne de la pechuga (RP, en \%). Previa disección de los músculos pectorales, se registró el peso de los mismos y por cálculo se obtuvo el rendimiento de carne de pechuga respecto a PL.

Rendimiento de carne de la pata-muslo (RPM, en \%). Previa disección de los músculos del muslo y la cadera, se registró el peso de los mismos y por cálculo se obtuvo el rendimiento de la carne de la pata muslo respecto a PL.

Porcentaje de grasa acumulada (G, en \%). Luego de la disección del panículo adiposo abdominal se registró el peso para calcular su rendimiento con relación a PL.

\section{Variables de calidad de carne}

Las muestras de los lotes de diferentes tratamientos y testigos fueron analizadas en el Laboratorio del Servicio de Tecnología de los Alimentos de la Facultad de Ciencias Veterinarias de la UNNE. Los atributos de calidad de carne a evaluar fueron:

Color: utilizando un colorímetro Minolta CR 400, por técnica CIElab (1986). Se determinó la luminosidad (L) y los índices $\mathrm{a}^{*} \mathbf{y} \mathbf{b}^{*}$.

Capacidad de retención de agua en \% (CRA), pérdidas por cocción en \% (PPC) y determinación de terneza $\left(\mathrm{kg} / \mathrm{cm}^{2}\right)$ : con cizalla Warnner Blatzer, por técnica de la AMSA (American Meat Science Asociation) y ASTM (American Society for Testing and Materials). 205.

El pH: por peachímetro pinchacarne marca Testo

Se realizó análisis estadístico mediante ANOVA para un diseño factorial $2 \times 4$ (dos sistemas de crianza por cuatro épocas del año), considerando un $5 \%$ como límite de significancia.

\section{RESULTADOS Y DISCUSIÓN}

Los resultados obtenidos en a los cuatro ensayos realizados, se consignan en las Tablas 1 y 2 .

Como se puede observar en Tabla 1 los pollos del grupo sin salida a parque (control) registraron menores valores para $\mathrm{pH}$ y pérdidas por cocción, lo cual sugiere un diferente equilibrio energético a favor de este grupo, resultando en un mayor acumulo de glucógeno, favoreciendo el proceso de transformación del músculo en carne $^{4,8}$.

Se demostraron también diferencias significativas para el valor $b^{*}$ en los pollos sin salida a parque. Por su parte, la terneza evidenció diferencias significativas con mayores valores en los pollos con acceso a parque (tratados), lo cual permite asumir un efecto de la actividad física sobre el acumulo de tejido conectivo en los músculos de este grupo experimental ${ }^{1,9}$.

Más allá de las diferencias significativas entre los grupos experimentales, para las características tecnológicas todos los valores se encuentran incluidos dentro de los rangos considerados normales para la carne de pechuga ${ }^{13,18}$.

En la Tabla 2 no se encontraron diferencias significativas en peso vivo, peso limpio y rendimiento de la canal. Sin embargo, se encontraron diferencias significativas en el porcentaje de grasa acumulada. Los pollos criados con acceso a parque registraron menor porcentaje de grasa acumulada junto a un mayor desarrollo de la pata muslo, lo cual sugiere que la mayor actividad física desarrollada por este grupo experimental dio por resultado una merma en la cantidad de grasa abdomi- 
nal, junto a un mayor incremento proteico en la pata muslo.

Ambos efectos observados en los pollos con acceso a parque, expresan un equilibrio energético diferente con derivación de energía al desarrollo del sistema de locomoción. Por otra parte, los pollos en confinamiento distribuyeron en forma diferente los nutrientes suministrados, resultando en mayor desarrollo de pechuga y acumulo graso abdominal.

Estos resultados confirmarían los hallazgos de otros autores que reportaron cambios en la composición corporal debido a factores de distinto origen, que ejercen influencia significativa tanto sobre la calidad, como sobre la composición de la canal: la edad, el sexo, la nutrición, la distribución de la grasa, la funcionalidad muscular, el estrés y otros ${ }^{14}$

En comparación con el sistema convencional en confinamiento los sistemas extensivos pueden disminuir las condiciones de estrés y aumentar la comodidad de las aves debido al desarrollo de la actividad física, lo que favorece el desarrollo osteomuscular de las patas junto a una mejora en la habilidad de caminar ${ }^{17}$.

El crecimiento del músculo es el principal determinante del rendimiento carnicero. La cantidad de carne producida está relacionada con el número de fibras musculares y el crecimiento de la fibra muscular individual en aves adultas ${ }^{1}$. La secuencia del desarrollo de los diversos músculos del cuerpo es indicativa de su importancia relativa en atender a las necesidades del animal. Así, el desarrollo precoz de los músculos de los miembros distales le confiere la movilidad requerida para la búsqueda de alimento ${ }^{11}$.

Estos resultados permiten concluir que el sistema de alojamiento afecta las características tecnológicas relacionadas con reserva energética muscular y síntesis de colágeno muscular. Asimismo, también sobre la composición de la canal, que refleja la diferente distribución de nutrientes mediante modificaciones en la actividad física desarrollada por las aves.

Tales hallazgos podrían considerarse propicios para fomentar nuevos modelos de producción que permitan la diversificación en contextos no tradicionales para la avicultura como actividad comercial.

\section{REFERENCIAS}

1. Andersen H, Oksbjerg N, Young J, Therkildsen $M$. 2005. Feeding and meat quality. A future approach. Meat Science 70: 543-554

2. Cepero BR. 2009. Avicultura alternativa. ¿Retorno al pasado, o un camino al futuro? Selecc Avic 51: 1, 73-79.

3. Cömert M, Şayan Y, Kirkpinar F, Bayraktar ÖH, Mert S. 2016. Comparison of carcass characteristics, meat quality, and blood parameters of slow and fast grown female broiler chickens raised in organic or conventional production system. Asian Austr J Anim Sci 29: 7, 987-997.
4. Equipo Técnico Biomin. 2019. Efecto del Digestarom ${ }^{\circledR}$ en la calidad de la carne de ave. Avi News, https://avicultura info/el-efecto-del-digestarom-en-la-calidad-de-la-carnede-ave.

5. EPSA (Estrategias Provinciales para el Sector Agroalimentario). 2009. Resolución del Ministerio de la Producción Ambiente $N^{\circ}$ 373/09, Provincia de Formosa, www. prosap.gov.ar

6. Fabregas CX. 2004. Bases normativas en la producción extensiva de pollos. Eurocarne (pp.0001-9). http://ddd.uab cat/pub/artpub/2004/69390/11322675n132p1.pdf

7. Huq F, Kabir M. 1999. The role of women in poultry development: Proshika Experiences. Dhaka, Bangladesh. In: http://www.fao.org/docrep/004/ac154/htm

8. Lebihan DE, Berri C, Baeza E, Millet N, Beaumont C. 2001. Estimation of the genetic parameters of meat characteristics and of their genetic correlations with growth and body composition in an experimental broiler line. Poultry Sci 80: 7, 839-843.

9. Moreno TR. 2005. Calidad de la carne de pollo. Selecciones avicolas. https://selecciones-avicolas.com/pdf-files/2005/7/1644.pdf

10. Mota RD et al. 2008. Welfare at slaughter of broiler chickens: a review. International Journal of Poultry Science 7 : $1,1-5$.

11. Lawrie RA. 1998. Ciencia de la carne, $3^{\text {ra }}$ ed, Edit. Acribia, Zaragoza, España.

12. Niranjan $\mathbf{M}$ et al. 2008. Comparative evaluation of production performance in improved chicken varieties for backyard farming. Intern J Poultry Sci 7: 1128-1131.

13. Özbek M, Petek M, Ardıçlı S. 2020. Physical quality characteristics of breast and leg meat of slow and fast growing broilers raised in different housing systems. Arch Anim Breed 63: 2, 337-344

14. Price JF, Dugan LR, Schweigert BS. 1994. Ciencia de la carne y de los productos cárnicos, $2^{\circ}$ ed., Acribia, Zaragoza.

15. Sarica $\mathbf{M}$ et al. 2014. Comparing slow-growing chickens produced by two and three way crossings with commercial genotypes. 2: Carcass quality and blood parameters. Europ Poult Sci 78, doi: 10.1399/eps.2014.30.

16. Sitio Argentino de Producción Animal. 2006. Sector Ganadero de la Provincia de Formosa - www.produccionanimal.com.ar

17. Wang KH, Shi SR, Dou TC, Sun HJ. 2009. Effect of a free-range raising system on growth performance, carcass yield, and meat quality of slow-growing chicken. Poultry Science 88: 2219-2223

18. Yagüe FA. 2017. Transformación del músculo en carne. Avi News, febrero 2017, https://avicultura.info/transformacion-del-musculo-carne/ 